\title{
The Surface Potential at the Air-water Interface Computed Using Density Functional Theory
}

\author{
Kevin Leung \\ Sandia National Laboratories, MS 1415, Albuquerque, NM 87185, kleung@sandia.gov
}

(Dated: September 18, 2018)

\begin{abstract}
An accurate prediction of the surface potential $(\phi)$ at the air-water interface is critical to calculating ion hydration free energies and electrochemical half-cell potentials. Using Density Functional Theory (DFT), model interfacial configurations, and a theoretical definition of $\phi$, we report a value of +3.63 volt at $0.92 \mathrm{~g} / \mathrm{cc}$ water density. A maximally localized Wannier function analysis confirms that $\phi$ is dominated by molecular quadrupole (or "spherical second moment") contributions. We find that the predicted surface potential depends on computational details, and conclude that standard DFT codes and the existing definition of $\phi$ may not yield surface potentials directly comparable to existing experiments.
\end{abstract}

"Surface potentials" (or "potentials of the phase") are invoked when a charged particle moves from one phase to another through their mutual interface. One of the simplest and most important examples pertains to the surface between air and water $\underline{\underline{1-16}}$ ("Air," "vacuum," and "vapor" will be used interchangeably in this work.) The electrostatic potential difference $(\phi)$ between an ion in vacuum at infinity and that ion in pure liquid water contributes a term $q \phi$ to the absolute hydration free energy $\left(\Delta G_{\text {hyd }}\right)$, where $q$ is the ionic charge. $\phi$ thus contributes to all aqueous media electrochemical half-cell potentials,$\frac{17,18}{1}$ which consist of $\Delta G_{\text {hyd }}$ plus the pertinent ionization potentials. Recent Density Functional Theory (DFT) calculations have yielded $\phi$ estimates for the air-water interface ${ }^{19}-23$ substantially larger in magnitude than classical force field predictions ${ }^{-5}-\underline{9}$ and experimental values, $12-16$ suggesting that the methodology and physical basis of such calculations should be re-examined.

We first focus on molecular simulation perspectives, $\underline{\underline{5}-\underline{\underline{9}}}$ deferring the question of what is measured in experiments to the concluding discussions. For this purpose, we consider a charge-neutral slab of salt-free liquid water. Using this kind of simulation cell, force field-based simulations (i.e., those not based on electronic structure calculations) have reported that $\phi$ depends on the water model used,,$\underline{-10}$ but that the widely applied SPC/E

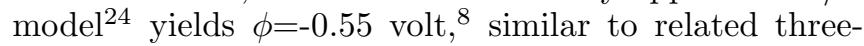
point point-charge water models, including polarizable ones $\stackrel{9}{\underline{9}}$

DFT takes into account electronic structure and molecular polarizabilites, and might be considered an improvement over non-polarizable water models. As will be shown, however, care must be exercised when interpreting the results based on the existing theoretical definition of the surface potential (see below) $\stackrel{5-9}{-9}$ A DFT work that extrapolates the highest occupied molecular orbital in gas phase ion/water-clusters to infinite cluster size has estimated that $\phi \sim 4$ volt $\underline{19}$ (in our notation).

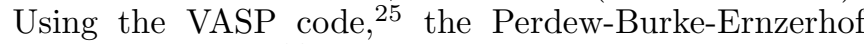
(PBE) functional,,$\frac{26}{}$ a bulk liquid simulation cell, and approximations for surface dipoles, we have deduced that $\phi=+4.05$ volt $\underline{21}$ By considering $\mathrm{p} K_{\mathrm{a}}$ and proton hy- dration free energies in DFT simulations, Cheng et al $\stackrel{22}{\underline{2}}$ have postulated that $\phi=+3.5$ volt. With explicit airwater interfaces, another DFT calculation has predicted $\phi=+3.1$ volt. $\stackrel{23}{ }$ In this work, we perform a DFT/PBE $\phi$ calculation with air-water interfaces to compare with previous predictions. We also use maximally localized Wannier functions 27 to analyze the results in terms of molecular contributions, which allows a detailed comparison of DFT and force-field based work: $\stackrel{1,5-11}{\underline{11}}$ Our work highlights the dependence of $\phi$ on computational details.

The theoretical $\phi$ is given by the difference in the average plateau values between the liquid and vacuum regions in $\bar{\phi}(z)$, where

$$
\bar{\phi}(z)=\left\langle\iint d x d y V(x, y, z) / A\right\rangle .
$$

$V(\mathbf{r})$ is the calculated electrostatic potential at point $\mathbf{r}$, $A$ is the lateral area of the simulation cell, $z$ is perpendicular to the interfaces, and the angular brackets denote statistical averaging. The analytic expressions derived for surface potentials, and formulas for dealing with long-range electrostatics within periodic boundary conditions in general, are based on purely coulombic, $1 / r$ potentials,,$\frac{17,28,29}{\text { Thus }}$

$$
V(\mathbf{r})=\int d \mathbf{r}^{\prime} \rho_{e}\left(\mathbf{r}^{\prime}\right) /\left|\mathbf{r}-\mathbf{r}^{\prime}\right|+\sum_{i} Z_{i} /\left|\mathbf{R}_{i}-\mathbf{r}\right| .
$$

Here $\rho_{e}(r)$ is the valence electron density, $\mathbf{R}_{i}$ is the position of pseudo-nuclei $i$, and $Z_{i}$ is the pseudo-nuclear charge in the pseudopotentials $(\mathrm{PP})$, with $Z_{\mathrm{O}}=+6|e|$ and $Z_{\mathrm{H}}=+|e|$. By default, VASP instead outputs the negative of Eq. 2 after replacing the last term with the entire local PP $U\left(\mathbf{R}_{i}, \mathbf{r}\right)$. The short range, non-coulombic contribution to $U\left(\mathbf{R}_{i}, \mathbf{r}\right)$ should be removed when computing $\phi$. This ambiguity arises from the use of PP's, and is absent in all-electron calculations.

100 water configurations are taken from a onenanosecond water-vapor interface molecular dynamics trajectory generated with $128 \mathrm{SPC} / \mathrm{E}$ water molecules in a $50.0 \times 12.5 \times 12.5 \AA^{3}$ simulation cell (see the Supporting Information, SI, for more details). $\bar{\phi}(z)$ is computed from these configurations using DFT/PBE and the 


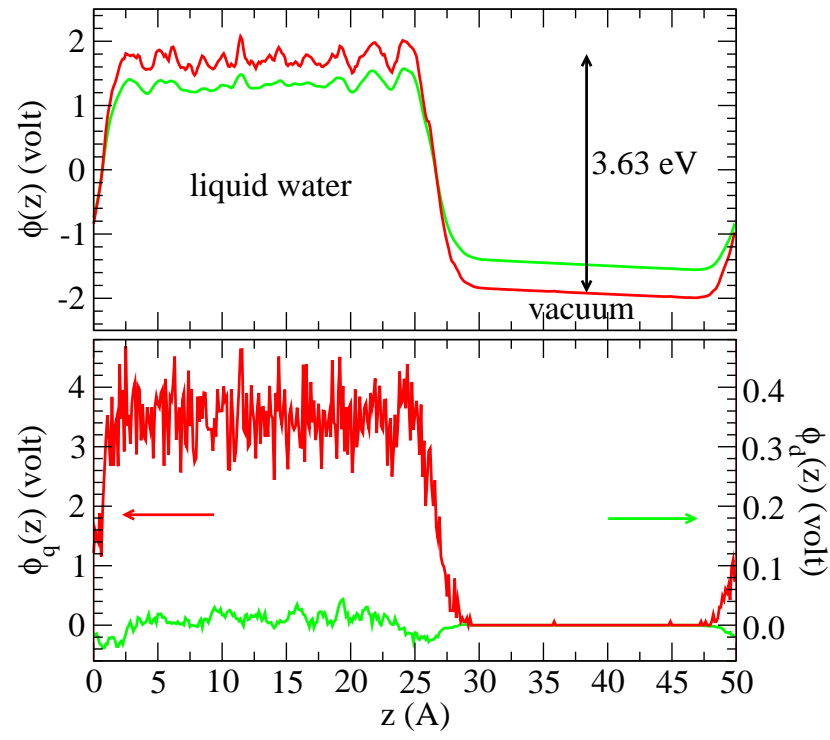

FIG. 1: (a) Electrostatic potential $(\bar{\phi}(z))$ computed perpendicular to water-vacuum interface direction $z$, averaged over the lateral ( $x$ - and $y$-) directions. The red and green lines represent predictions using purely coulomb interactions (Eq. 2) and $U\left(\mathbf{R}_{i}, \mathbf{r}\right)$ (see text). $\int_{z} \bar{\phi}(z)=0$ over the simulation cell. (b) Quadrupole (red) and dipole (green) contributions to $\bar{\phi}(z)$. The latter has its finite electric field removed and is then expanded ten-fold; the quadrupole component contains more spatial noise than $\bar{\phi}(z)$.

Eq. 2 definition. Figure 17 shows that $\phi$ averages to $+3.63 \pm 0.04$ volt when referenced to vacuum. If we had used the VASP default and included the short range part of the PP, $\phi$ would become +2.8 volt instead. Clearly, the details of the algorithm strongly affect the results. The vacuum region exhibits a small, 0.005 volt $/ \AA$ electric field, indicating that a small but finite net average dipole moment persists in the water slab due to insufficient statistical sampling.

$\phi$ can be rigorously decomposed into quadrupole and dipole components. $\frac{1,7,8,29}{1 n}$ water, they can be written

$$
\begin{aligned}
\phi & =\phi_{q}+\phi_{d} \\
& =\bar{\phi}_{q}\left(z_{\text {water }}\right)-\bar{\phi}_{q}\left(z_{\text {air }}\right)+\bar{\phi}_{d}\left(z_{\text {water }}\right)-\bar{\phi}_{d}\left(z_{\text {air }}\right) \\
\bar{\phi}_{q}(z) & =\left\langle-(2 \pi / 3 A) \int d \mathbf{r} \delta\left(z-R_{m, z}\right) \sum_{m} \rho^{m}(\mathbf{r})\left(\mathbf{r}-\mathbf{R}_{m}\right)^{2}\right. \\
\bar{\phi}_{d}(z) & =\left\langle 4 \pi / A \int d \mathbf{r} \sum_{m} \rho^{m}(\mathbf{r})\left(r_{z}-R_{m, z}\right) \Theta\left(z-R_{m, z}\right)\right\rangle .
\end{aligned}
$$

Here $\rho^{m}(\mathbf{r})=\rho_{e}^{m}(\mathbf{r})+\sum_{i} Z_{i}^{m} \delta\left(\mathbf{r}-\mathbf{R}_{i}\right)$ is the charge density of molecule $m$ with all electrons and nuclei $i$ residing on $m, \mathbf{R}_{m}$ is the molecular center (oxygen atom in the case of water), and $\Theta$ is the Heaviside function. Calculating $\phi_{d}$ requires an interfacial geometry. As an interface was not present in Ref. 21, $\phi_{d}$ was approximated using the $\mathrm{SPC} / \mathrm{E}$ water value $(+0.21$ volt $) \underline{\underline{8}}$

In contrast, $\phi_{q}$ can be computed in a bulk liquid simu- lation cell, with electron density demarcated into molecular contributions using maximally localized Wannier functions $\frac{20.27}{2}$ Predicted to be +3.85 volt at $1.0 \mathrm{~g} / \mathrm{cc}$ water density $\stackrel{21}{1}$ this VASP/PBE $\phi_{q}$ is larger in magnitude than and opposite in sign to that for $\mathrm{SPC} / \mathrm{E}$ water $\left(\phi_{q}=-0.76\right.$ volt) because of differences in the charge distributions (Fig. 2). Using the $\mathrm{O}$ atom as the molecular center, only the partial positive charges on the H-sites of SPC/E water contribute to Eq. 4 yielding a negativedefinite $\phi_{q}$. For VASP/PBE, Eq. 4 is instead dominated by the valence electron cloud surrounding the O-nuclei and $\phi_{q}$ changes sign.

To further analyze the results, we also use maximally localized Wannier functions to decompose the interfacial $\bar{\phi}(z)$ (Fig. 1a) into quadrupole and dipole contributions (Fig. 1b). In the bulk liquid region, defined as $5 \AA<z<20 \AA, \phi_{q}$ averages to $+3.50 \pm 0.01$ volt. This differs from the +3.85 volt derived indirectly (see the SI) because unlike bulk SPC/E water calculations,,$\underline{24}$ our small lateral simulation cell dimensions dictate a small Lennard-Jones cut-off distance for SPC/E water, which reduces the density of the bulk liquid region to $0.92 \mathrm{~g} / \mathrm{cc}$. Equation 4 implies that $\phi_{q}$ is proportional to the liquid water density $: 1,7$ Consistent with this formula, the $8 \%$ reduction in $\rho_{\text {water }}$ and the $9.6 \%$ decrease in $\phi_{q}$ compared to the indirect calculation conducted at $1.0 \mathrm{~g} / \mathrm{cc}$ water density indeed track each other. We have not used PBE-based molecular dynamics to generate water slabs partly because PBE exhibits water densities that deviate even more strongly from experiments $\underline{\underline{30}}$ The +3.1 volt DFT value reported previously ${ }^{23}$ likely also reflects the low DFT water density present in that work.

The small cell also affects $\phi_{d}$. After removing the finite average electric field in the 100 configurations selected, the SPC/E $\phi_{d}$ amounts to +0.009 volt, strongly reduced from the $\phi_{d}=+0.21$ volt computed in a larger box. ${ }^{-}$DFT/PBE applied to these SPC/E interfacial configurations tracks $\mathrm{SPC} / \mathrm{E}$ results, giving $\phi_{d}=+0.012 \pm 0.008$ volt (Fig. 1b). $\phi_{d}$ and $\phi_{q}$ thus add to +3.52 volt, which is consistent with the +3.63 volt obtained directly using Eq. 1. The small discrepancy may arise from the fact that the interfacial simulation cell has prevented the extrapolation of Wannier estimates of $\phi_{q}$ (3)infinite box size as was done in Ref. 20.

The term "quadrupole moment" used to describe $\phi_{q}$ . (2:) Eq. 4 is taken from the liquid state literature, $1,4,7,8$ "radial" and "spherical second moment" 20,29 have been kssed elsewhere. Equation 4 contains the trace of quadrupole tensor, typically set to zero in multiple expansions and does not contribute to real-space electrostatic interactions ${ }^{31}$ As an example, the atoms in a neon solid are largely spherically symmetric and exhibit only small multipole (including off-diagonal quadrupole) moments, but at the equilibrium density of $1.444 \mathrm{~g} / \mathrm{cc}$ at its melting point, our DFT/PBE/PP $\phi_{q}$ estimate for solid neon still amounts to 3.6 volt.

This neon example emphasizes that the theoretical $\phi$ in Eq. 1. computed using the PBE functional, is not a 


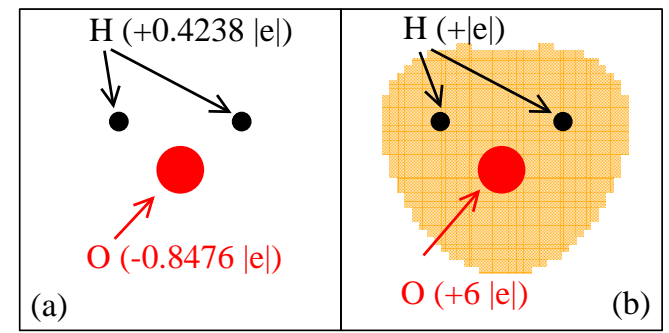

FIG. 2: Charge distributions. (a) SPC/E water model; (b) projection of DFT/PBE water, using pseudopotentials. O, $\mathrm{H}$, and electron density exceeding $0.05|e| / \AA^{3}$ are depicted in red, black, and orange respectively.

physical quantity. Indeed, the PP used omits oxygen core $1 s$ electron contributions (see the SI); otherwise the magnitude of $\phi$ would be even larger (Eq. 4) ${ }^{32}$ This theoretical $\phi$ is however critical for DFT-based molecular dynamics calculations of ion hydration free energies $\left(\Delta G_{\text {hyd }}\right) \stackrel{21,22}{\stackrel{2}{2}}$ The reason, familiar in the classical force field literature, $1,5-\underline{\underline{7}}, 9,11$ is that $\Delta G_{\mathrm{hyd}}$ at infinite dilution are most conveniently computed as intrinsic ion hydration free energies calculated in bulk-water boundary condition simulation cells using Ewald summation, with the non-ion-specific pure water "surface potential" contribution $q \phi$ added during post-processing. Ewald technques arbitarily set the average electrostatic potential to zero over the bulk-water simulation cell. The "true" (but computation protocol-specific) average electrostatic potential is restored by referencing the liquid region plateau $\bar{\phi}(z)$ value to vacuum. This of course involves a rigid shift equal to $\phi$, which must be obtained using the same definition and pseudopotentials applied in intrinsic ion hydration calculations. In DFT calculations, $\phi$ and the intrinsic ion hydration free energy (the latter through Ewald summation conventions) both contain large, equal but opposite contributions from the water atomic core regions (Fig. 20) $\frac{20}{2}$ even though ions do not penetrate into water nuclei. $\phi$ must be on the order of +4 volt to yield $\Delta G_{\text {hyd }}$ comparable to experimental data. 21 If the VASP/PBE $\phi$ were -0.55 volt like in SPC/E water, hydration of $\mathrm{Cl}^{-}$ would have been endothermic and unphysical $\stackrel{20}{\underline{20}}$ Work function calculations in metals take advantage of a similar cancellation of ambiguities $\underline{33}^{33}$

We have so far side-stepped the issue of comparison with experiments. To the extent that the theoretical $\phi$ is mainly of interest for computing the absolute hydration free energies of ions at infinite dilution, it can be treated as a method- or force field-dependent entity. Indeed, it has been argued that the Galvani potential difference between two phases is extremely difficult to measure, although possible in principle $\stackrel{\underline{1}}{=} \phi$ also depends non-trivially on the salt present at the surface $\stackrel{\underline{3}}{\frac{3}{3}}$ To our knowledge, in

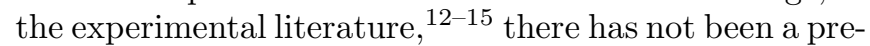
cise definition of $\phi$ in terms of microscopic (i.e., electronic and ionic) properties $\stackrel{2}{2}$ To the extent that this quantity has been indirectly measured at the air-water interface, early reported values strongly varied in magnitude and sign. ${ }^{13}$ Several post-1970 experimental values are in better agreement with each other $\stackrel{12,14}{1}$ yielding +0.025 to 0.16 volt values (see Ref. 16, Table 1). They are also in reasonable agreement with the dipole contribution $\phi_{d}$ of the $\mathrm{SPC} / \mathrm{E}$ water model,,$\frac{8}{}$ which raises the intriguing point that these measurements may predominantly reflect $\phi_{d}$. However, these experimental values cannot be directly used to help calculate DFT-based ion hydration free energies for reasons discussed above.

It may become possible to establish unambiguous experimental surface potentials in the future. Here we confine ourselves to the observation that, just as the theoretical $\phi$ depends on the method used, the measured $\phi$ may be sensitive to experimental details. (1) Surface sensitive spectroscopic measurements may yield values for $\phi$ that most closely match all-electron (frozen oxygen $1 s$ electrons or otherwise) DFT results. They amount to using as probes test particles that are point charges and do not exhibit many-Fermion effects. Proposed measurement of $\phi$ using electron reflectivity $\underline{\underline{1}}$ with $1-10 \mathrm{keV}$ beams should also probe the nuclear region, although many-Fermion effects may arise. (2) If ions are used as experimental probes, e.g., by considering ion hydration free energies, the solvent atomic nuclear regions are not sampled. These regions are mainly responsible for the large and positive $\phi$ in DFT calculations. Therefore a $\phi$ value different from case (1) above should emerge. It is conceivable that $\phi$ predicted using classical force fields, which do not contain distributed charges in atomic nuclear regions, may be more appropriate here. (3) In electrochemical measurements,, $12-16$ electrons are added to/removed from electrodes while ions enter/depart electric double layers. Hence a mixture of electrons and ions are implicitly used as probes. In light of the present work, the results should contain $\phi$ contributions from atomic nuclear regions of electrodes but not from water.

In conclusion, using $\mathrm{SPC} / \mathrm{E} \quad \mathrm{H}_{2} \mathrm{O}$ model-derived interfacial configurations, we have shown that our DFT/PBE pseudopotential calculation directly yields a $+3.63 \pm 0.04$ volt surface potential when we use the theoretical definition of $\phi$ and a water slab with interior density of $0.92 \mathrm{~g} / \mathrm{cc}$. Maximally localized Wannier function analysis confirms that this value is dominated by the density-dependent molecular quadrupole (or "spherical second moment") contribution $\phi_{q}$, consistent with previous calculations $\stackrel{20,21}{=}$ This suggests that the most robust way to estimate the dominant $\phi_{q}$ term in DFT calculations is to use a bulk liquid water simulation cell at $1.0 \mathrm{~g} / \mathrm{cc}$ density. The DFT surface dipole component of $\phi$ is not accurately determined due to finite size effects, but it tracks the SPC/E value for this simulation cell. This theoretical $\phi$ is not a physical quantity, in the sense that it is not what is measured in existing electrochemical experiments $\frac{12}{2}$ But it is critical for calculatin ion hydration free energies and modeling electrochemical 
half cell reactions. In general, $\phi$ computed in DFT calculations are sensitive to simulation details and may not be directly compared to experiments.

We thank Chris Mundy, Shawn Kathmann, Lawrence Pratt, Susan Rempe, Michiel Sprik, Don Truhlar, and Graham Yelton for interesting discussions, but stress that not all of them may agree with the perspectives expressed herein. We also thank Dr. Kathman for sharing
Ref. 23 prior to publication. This work was supported by the Department of Energy under Contract DE-AC0494AL85000. Sandia is a multiprogram laboratory operated by Sandia Corporation, a Lockheed Martin Company, for the U.S. Department of Energy.

Supporting Information Available: This material is available free of charge via the Internet at http://pubs.acs.org
1 Pratt, L.R. Contact Potentials of Solution Interfaces: Phase Equilibrium and Interfacial Electric Fields. J. Phys. Chem. 1992, 96, 25.

2 Guggenheim, E.A. The Conceptions of Electrical Potential Difference Between Two Phases and the Individual Activities of Ions. J. Phys. Chem. 1929, 33, 842.

3 Zhou, Y.Q.; Stell, G.; Friedman, H.L. Note on Standard Free Energy of Transfer and Partitioning of Ionic Species between 2 Fluid Phases. J. Chem. Phys. 89, 3836 (1988).

4 Stillinger, F.H.; Ben-Naim, A. Liquid-Vapor Interface Potential for Water. J. Chem. Phys. 1967, 47, 4431.

5 Wilson, M.A.; Pohorille, A.; Pratt, L.R. Molecular Dynamics of the Water Liquid-Vapor Interface. J. Phys. Chem. 1987, 91, 4873.

${ }^{6}$ Wilson, M.A.; Pohorille, A.; Pratt, L.R. Surface Potential of the Water Liquid-Vapor Interface. J. Chem. Phys. 1988, 88,3281 .

7 Wilson, M.A.; Pohorille, A.; Pratt, L.R. Comment on "Study on the Liquid-Vapor Interface of Water. 1. Simulation Results of the Thermodyanmic Properties and Orientational Structure." J. Chem. Phys. 1989, 90, 5211.

8 Sokhan, V.P.; Tildesley, D.J. The Free Surface of Water: Molecular Orientation, Surface Potential, and Nonlinear Susceptibility. Mol. Phys. 1997, 92, 625.

9 Dang, L.X.; Chang, T.-M. Molecular Mechanism of Ion Binding to the Liquid/Vapor Interface of Water. J. Phys. Chem. B 2002, 106, 235.

10 Brodskaya, E.N.; Zakharov, V.V. Computer Simulation Study of the Surface Polarization of Pure Polar Liquids. J. Chem. Phys. 1995, 102, 4595.

11 Kastenholz, M.A.; Hünenberger, P.H. Computation of Methodology-independent Ionic Solvcation Free Energies from Molecular Simulations. 1. The Electrostatic Potential in Molecular Liquids. J. Chem. Phys. 2006, 124, 124106.

12 Fawcett, W.R. The Ionic Work Function and its Role in Estimating Absolute Electrode Potentials. Langmuir 2008, 24, 9868.

13 Parfenyuk, V.I. Surface Potential at the Gas-Aqueous Solution Interface. Colloid J. 2002, 64, 588.

14 Gomer, R.; Tryson, G. An Experimental Determination of Absolute Half-cell emf's and Single Ion Free Energies of Solvation. J. Chem. Phys. 1977, 66, 4413.

15 Pethica, B.A. Are Electrostatic Potentials between Regions of Different Chemical Composition Measurable? The Gibbs-Guggenheim Principle Reconsidered, Extended, and its Consequences Revisited. Phys. Chem. Chem. Phys. 2007, 9, 6253.

16 Paluch, M. Electrical Properties of Free Surface of Water and Aqueous Solutions. Adv. Coll. Interface Sci. 2000, 84, 27 , and references therein.

17 Jaque, P.; Marenich, A.V.; Cramer, C.J.; Truhlar, D.G. Computational Electrochemistry: The Aqueous $\mathrm{Ru}^{3+} \mid \mathrm{R}^{2+}$
Reduction Potential. J. Phys. Chem. C 2007, 111, 5783.

18 VandeVondele, J.; Ayala, R.; Sulpizi, M.; Sprik, M. Redox Free Energies and One-electron Energy Levels in Density Functional Theory Based Ab Initio Molecular Dynamics. J. Electroanal. Chem. 2007, 607, 113.

19 Hunt, P.; Sprik, M. On the Position of the Highest Occupied Molecular Orbital in Aqueous Solutions of Simple Ions. Chem. Phys. Chem 2005, 6, 1805.

${ }^{20}$ Leung, K.; Marsman, M. Energies of Ions in Water and Nanopores within Density Functional Theory. J. Chem. Phys. 2007, 127, 154722.

${ }^{21}$ Leung, K.; Rempe, S.B.; von Lilienfeld, O.A. Ab Initio Molecular Dynamics Calculations of Ion Hydration Free Energies. J. Chem. Phys. 2009, 130, 204507.

22 Cheng, J.; Sulpizi, M.; Sprik, M. Redox Potentials and $\mathrm{p} K_{a}$ for Benzoquinone From Density Functional Theory Based Molecular Dynamics. J. Chem. Phys. 2009, 131, 154504.

${ }^{23}$ Kathmann, S.M.; Kuo, I.-F.W.; Mundy, C.J. Electronic Effects on the Surface Potential at the Vapor-Liquid Interface of Water. J. Am. Chem. Soc. 2008, 130, 16556; 2009, 131,17522 (the value is revised to +3.1 volt).

24 Berendsen, H.J.C.; Grigera, J.R.; Straatsma, T.P. The Missing Term in Effective Pair Potentials. J. Phys. Chem. 1987, 91, 6269 .

25 Kresse, G.; Furthmüller, J. Efficienct Iterative Schemes for Ab Initio Total-Energy Calculations Using Using a Planewave Basis Set. Phys. Rev. B 1996, 54, 11169.

26 Perdew, J. P.; Burke, K.; Ernzerhof, M. Generalized Gradient Approximation Made Simple. Phys. Rev. Lett. 1996, 77,3865 .

27 Marzari, N.; Vanderbilt, D. Maximally Localized Generalized Wannier Functions for Composite Energy Bands. Phys. Rev. B 1997, 56, 12847.

28 Hummer, G.; Pratt, L.R.; Garcia, A.E. Free Energy of Ionic Hydration. J. Phys. Chem. 1996, 100, 1206.

29 Saunders, V.R.; Freyria-Fava, C.; Dovesi, R.; Salasco, L.; Roetti, C. On the Electrostatic Potential in Crystalline Systems where the Charge Density is Expanded in Gaussian Functions. Mol. Phys. 1992, 77, 629.

30 McGrath, M.J.; Siepmann, J.I.; Kuo, I.-F.W.; Mundy, C.J. Vapor-liquid Equilibria of Water from First Principles: Comparison of Density Functionals and Basis Sets. Mol. Phys. 2006, 104, 3619.

31 Jackson, J.D. Classical Electrodynamics (Wiley, 1975), Ch. 4.

32 The effect of replacing point charges in water models with distributed charges has also been discussed in Ref. 6.

33 Lang. N.D.; Kohn, W. Theory of Metal Surfaces - Work Function. Phys. Rev. 1971, 3, 1215. 